\title{
ORIGINAL
}

\section{ABSORCIÓN SISTÉMICA DE FLÚOR EN NIÑOS SECUNDARIA AL CEPILLADO CON DENTÍFRICO FLUORADO}

\author{
José-María García-Camba de la Muela (1), Felisa García-Hoyos (1), Margarita Varela Morales (2) \\ y Ángel González Sanz (3)
}

(1) Facultad de Ciencias de la Salud. Departamento de Odontología. Universidad Europea de Madrid.

(2) Servicio de Ortodoncia. Fundación Jiménez Díaz. Madrid.

(3) Facultad de Ciencias de la Salud. Odontología. Universidad Rey Juan Carlos. Madrid.

\section{RESUMEN}

Fundamento: El efecto del flúor en la prevención de la caries está vinculado con su acción tópica. Cuando es ingerido, su efecto anticaries es escaso y, además, puede llegar a provocar efectos indeseables, entre ellos la fluorosis dental en el niño. El cepillado con pastas fluoradas es un procedimiento adecuado para vehicular el flúor tópico, pero en niños pequeños comporta riesgo de deglución inadvertida. El objetivo de este estudio es determinar la cantidad de flúor que aparece en orina después del cepillado con dentífrico fluorado.

Métodos. En una muestra de 115 niños de 5 a 8 años seleccionados aleatoriamente se analizaron los niveles de $\mathrm{pH}$, creatinina, y flúor en la orina excretada antes del cepillado con dentífrico fluorado y 2 horas después. Se utilizó la razón flúor/creatinina $(\mathrm{F} / \mathrm{Cr}$ ) para minimizar las variaciones del flúor en orina debidas a las diferentes tasas de secreción. Para el análisis estadístico se utilizaron la media, la desviación típica $\mathrm{y}$ el test $\mathrm{T}$ para las variables con distribuciones normales y pruebas no paramétricas para las variables con distribución no normal.

Resultados: La cantidad media de pasta utilizada fue $1.192 \mathrm{mg}$ (DT:421). Las variables $\mathrm{pH}$ y creatinina ofrecieron valores similares en las dos muestras de orina analizadas $(\mathrm{pH}$ : $6,35$ y 6,36 ; creatinina: $0,76 \mathrm{~g} / \mathrm{l}$ y $0,81 \mathrm{~g} / \mathrm{l})$. Sin embargo, la razón $\mathrm{F} / \mathrm{Cr}$ aumentó significativamente tras el cepillado, pasando de 0,61 a $1,25 \mathrm{mg} / \mathrm{g}$.

Conclusiones: La utilización de un dentífrico fluorado se asocia con un aumento significativo de la concentración de flúor en la orina del niño.

Palabras clave: Flúor. Fluoruros. Creatinina. Salud bucal. Infancia.

Correspondencia:

José-María García-Camba de la Muela

C/ Donoso Cortes $80 \mathrm{~A}$

28015 Madrid

Correo electrónico: jmgcam@gmail.com

\section{ABSTRACT}

\section{Demonstration of Fluoride Systemic Absorption Secondary to Toothbrusing with Fluoride Dentifrice in Children}

Background: The effect of the fluoride in the caries prevention is strongly linked to its topical action. When the fluoride is ingested, its anticaries effect is weak and, in addition, it can cause side effects such as dental fluorosis in children. The toothbrushing with fluoride dentifrice is a suitable procedure to provide the topical fluoride, but in small children there is risk of inadvertent swallowing. The main goal of this study is to quantify the fluoride levels in urine after toothbrushing with fluoride dentifrice.

Methods: A sample of 115 children between 5 to 8 years was randomly selected. The $\mathrm{pH}$, as well as creatinine and fluoride levels were analyzed in the urine excreted before and two hours after toothbrushing with fluoride dentifrice. The fluoride/creatinine $(\mathrm{F} / \mathrm{Cr})$ ratio was used to minimize the variations of the fluoride levels in urine due to the different rates of secretion. For the statistical analysis, the average, the standard deviation and the $\mathrm{T}$ test were used for the variables with normal distributions, and nonparametric tests were applied for the variables with non normal distribution.

Results: The average amount of dentifrice was $1192 \mathrm{mg}$ (SD: 421). The variables $\mathrm{pH}$ and creatinine showed similar values in the two samples of urine analyzed $(\mathrm{pH}$ : 6,35 and 6,36; creatinine: $0,76 \mathrm{~g} / \mathrm{l}$ and $0,81 \mathrm{~g} / \mathrm{l})$. Nevertheless, the $\mathrm{F} / \mathrm{Cr}$ ratio increased significantly after toothbrushing, ranging from 0.61 to $1.25 \mathrm{mg} / \mathrm{g}$.

Conclusions: The use of fluoride dentifrice is related to a significant increase of the fluoride concentration in urine in children.

Key words: Fluorine. Fluor Protector [Substance Name]. Fluorides. Creatinine. Oral health. Childood. 


\section{INTRODUCCIÓN}

Como resultado de las actuales estrategias preventivas se ha producido un descenso considerable en la prevalencia de caries en la mayoría de los países desarrollados $^{1-3}$. La razón más aceptada para explicar este descenso es el uso generalizado de flúor en todas sus modalidades, cuyos efectos beneficiosos en la prevención de la caries dental están ampliamente documentados desde que comenzó a utilizarse hace aproximadamente sesenta años ${ }^{4}$.

En el momento actual, el flúor con fines preventivos se vehicula a través de aguas fluoradas, tabletas, gotas, colutorios, geles, barnices y, en especial, dentífricos fluorados, que han demostrado tener el máximo efecto anticaries ${ }^{5,6}$.

La administración de flúor como elemento preventivo es posiblemente el procedimiento más eficaz para evitar la caries dental, pero se debe considerar el riesgo previo de caries del individuo receptor de esta medida. La Conferencia de Toronto en $1998^{7}$, desaconseja los suplementos fluorados, a excepción del dentífrico fluorado, en los niños con escaso riesgo de caries. La recomendación para niños de alto riesgo mayores de 3 años que no residen en zonas de aguas fluoradas es la administración individualizada de 0,50 a 1,00 mg de FNa en forma de tabletas orales, en algunos casos suplementada con otros productos fluorados de acción tópica.

A pesar de esta recomendación, la tendencia en las últimas décadas ha sido la sobredosificación de flúor, en muchos casos condicionada por la aplicación simultánea de múltiples tratamientos fluorados. Estas pautas, justificadas en individuos con riesgo alto de caries, podrían provocar fluorosis dental en niños pequeños, una anomalía del desarrollo del esmalte que se produce como consecuencia de la excesiva ingestión de flúor durante la fase de formación dentaria ${ }^{8-11}$. Además, hay que considerar el gasto metabólico innecesario al que se somete al organismo al tener que metabolizar más flúor del que sería suficiente para cubrir los objetivos preventivos.

En cualquier caso, el cepillado diario con dentífrico fluorado es muy beneficioso para los dientes del niño. El flúor vehiculado en el dentífrico, actuando tópicamente, aumenta la remineralización del esmalte, disminuye su desmineralización y ejerce una eficaz acción antibacteriana, fundamentalmente contra las bacterias más cariogénicas como son los estreptococos y los lactobacilos.

Sin embargo, está comprobado que el cepillado de dientes conlleva la deglución inadvertida, o incluso voluntaria, de cierta cantidad de dentífrico ${ }^{12,13}$, un hecho que puede adquirir particular relevancia en los niños más pequeños ${ }^{14,15}$. Además, el flúor contenido en la pasta que ingresa en el organismo a nivel sistémico proporciona muy poco beneficio como elemento preventivo de la caries ${ }^{16}$.

El 75-90 \% del flúor ingerido es absorbido pasivamente en estómago y duodeno ${ }^{17}$. Esta absorción es rápida y depende del pH del entorno ${ }^{18}$. El $50 \%$ de la absorción se produce en los primeros 30 minutos $^{19}$ y la concentración plasmática máxima del flúor se alcanza a los 60 minutos $^{20}$.

La eliminación del flúor absorbido se realiza casi exclusivamente por vía renal. El aclaramiento renal de flúor en niños es de $45 \mathrm{ml} / \mathrm{min}^{21}$. El porcentaje de flúor filtrado que es reabsorbido oscila entre el $10 \%$ y el $90 \%$, dependiendo en gran medida del $\mathrm{pH}$ del fluido tubular ${ }^{22}$. Así, los factores que afecten al $\mathrm{pH}$ de la orina pueden alterar la excreción urinaria del flúor ${ }^{23}$. La excreción del flúor a través de la orina aumenta des- 
pués de la ingestión ${ }^{20}$, siendo máxima a las 1,5-3 horas para disminuir después rápidamente 24 .

Aunque la absorción sistémica del flúor administrado con fines preventivos puede comportar riesgos como la intoxicación aguda ${ }^{25}$ y la fluorosis dental, son muy pocos los estudios publicados en los que se analiza específicamente la eliminación de flúor en orina tras el cepillado con un dentífrico fluorado como expresión de dicha absorción sistémica. En la mayoría de esos estudios se analizan los niveles de flúor en orina en relación con el flúor eventualmente ingerido a través de todos los vehículos simultáneamente: dieta, aguas fluoradas y otros suplementos además del dentífrico ${ }^{13,14}$ y las determinaciones se llevan a cabo en orina de 24 horas $^{26}$. No obstante, también puede recurrirse a la cuantificación del flúor en muestras de orina puntuales, recogidas en el periodo de tiempo en el que se espera encontrar el pico máximo de excreción, es decir, aproximadamente a las dos horas de la exposición al flúor. Cuando se realiza este tipo de análisis, y para reducir al mínimo las variaciones del flúor en orina debidas a las diferentes tasas de excreción, se determinan los valores de la razón flúor $(\mathrm{mg}) /$ creatinina $(\mathrm{g})(\mathrm{F} / \mathrm{Cr})$, los cuales se consideran equivalentes a los encontrados en muestras de orina de 24 horas cuando las concentraciones de creatinina son normales (entre 0,5 y 3,0 $\mathrm{g} / 1)^{27-31}$.

El objetivo fundamental de este trabajo es determinar las concentraciones de flúor en la orina antes y después del cepillado dental con un dentífrico fluorado.

\section{SUJETOS Y MÉTODOS}

El estudio fue realizado en una muestra de escolares del colegio público San José de Las Matas, en Madrid, una localidad cuyas aguas de consumo no están fluoradas y presentan unas concentraciones de flúor inferiores a $0,3 \mathrm{ppm}$.

Previamente al trabajo de campo, para determinar el tamaño de la muestra mediante análisis de varianzas, se realizó un estudio piloto con 10 individuos (5 niños y 5 niñas). La muestra definitiva quedó constituida por 115 escolares con edades comprendidas entre 5 y 8 años. De éstos, 100 (57 niños y 43 niñas) fueron asignados al "grupo de estudio", que iba a cepillarse con dentífrico fluorado y los 15 restantes (7 niños y 8 niñas), que no se iban a cepillar los dientes, se consideraron "grupo de referencia". Ambos grupos fueron elegidos aleatoriamente entre todos los niños susceptibles de participar en el estudio.

Los criterios de inclusión fueron: contar con el consentimiento informado de padres o tutores, que el niño efectuara el cepillado dental habitualmente sin ayuda sirviéndose por sí mismo el dentífrico y, por último, que aportara suficiente cantidad de orina como para poder ser analizada. Los criterios de exclusión comprendían la existencia de enfermedades renales y otras enfermedades sistémicas.

En el centro escolar los niños fueron convocados en grupos de aproximadamente 15 individuos. Se les preguntó si ellos mismos realizaban su cepillado en casa, descartando a los que se cepillaban habitualmente con ayuda y a aquéllos a los que la pasta les era suministrada por adultos. Seguidamente se entregó a cada niño un frasco estéril y se le instruyó para que orinara en su interior. Después de haber recogido la primera muestra de orina se distribuyó a cada uno de los niños que iban a realizar el cepillado un cepillo dental y un tubo de dentífrico y se les pidió que colocaran la pasta sobre el cepillo tal y como lo hacían habitualmente en casa. Este procedimiento se realizó separando suficiente- 
mente a los niños para evitar que se influenciaran durante el mismo.

El dentífrico utilizado fue Colgate "Bugs Bunny" ${ }^{\circledR}$, con un contenido de $\mathrm{FNa}$ de 1.100 ppm. Otros componentes de esta pasta son: agua, sorbitol, sílice hidratada, pirofosfato tetrasódico, celulosa, lauril sulfato de sodio, sacarina sódica, glicerina y excipientes. Una vez colocada la pasta en el cepillo se cuantificó la cantidad utilizada por cada niño.

En los trabajos publicados en los que se realizan este tipo de mediciones se emplean métodos muy diversos de pesaje, que van desde mediciones individuales muy estrictas hasta la utilización de valores medios a partir de las cantidades de pasta utilizadas globalmente por los grupos experimentales Para realizar este trabajo se decidió emplear un sistema que permitiera estudiar individualmente a cada niño. Se optó por este sistema aún asumiendo los inconvenientes que comporta la cuantificación de la pasta dental mediante categorías, pues permitía extraer conclusiones sobre cada individuo en cuanto a la influencia de la dosis de dentífrico utilizada sobre el flúor excretado en la orina.

El método de evaluación seguido fue el siguiente: antes del estudio los investigadores habían realizado en el laboratorio seis pesajes independientes del cilindro de dentífrico que ocupaba respectivamente la totalidad del cabezal de seis cepillos idénticos a los que posteriormente habrían de utilizar los niños en el estudio. Para ello se empleó una balanza de Ohaus TS120 AS con una precisión de $1 \mathrm{mg}$. El valor medio de dichos pesajes fue 2.031 mg. A continuación se dividió virtualmente el cabezal del cepillo en 6 segmentos de una longitud aproximada de 3,3 $\mathrm{mm}$ y se calculó el peso medio de la pasta que ocuparía cada segmento, siendo el resultado $339 \mathrm{mg}$. De ese modo, se asu- mió que el volumen de pasta correspondiente a $3,3 \mathrm{~mm}$ de longitud del cilindro de dentífrico aplicado sobre el cepillo pesaría $339 \mathrm{mg}$.

Sobre esa base, para evaluar el peso de pasta utilizada por cada niño de la muestra experimental, se utilizó una regleta graduada con seis segmentos de 3,3 mm cada uno, lo que permitió clasificar a los niños en seis categorías, en función de su correspondiente "dosis de dentífrico".

Previamente al momento del cepillado, una vez que el niño había colocado el dentífrico en el cepillo, un miembro del grupo de estudio medía con la regleta la longitud del cilindro correspondiente, puntuando el resultado de 1 a 6 . La cantidad de pasta utilizada se analizó por edades, dividiendo la muestra en dos grupos: uno integrado por los niños de 5-6 años y el otro por los niños de 7-8 años.

A los niños que formaban parte del grupo de referencia que no se habían cepillado los dientes se les asignó la puntuación 0 .

A continuación se pedía a los niños del grupo de estudio que se cepillaran tal y como lo hacían habitualmente en casa durante un periodo cronometrado de 2 minutos y que después se enjuagaran con agua. Seguidamente los niños reanudaban sus actividades académicas con la instrucción de no comer ni beber nada hasta ser nuevamente convocados. Al cabo de dos horas se tomaba una segunda muestra de orina en cada niño.

En las distintas muestras de orina se determinaron el pH y las concentraciones de flúor y creatinina. Las medidas del $\mathrm{pH}$ se realizaron mediante el método potenciométrico, las del flúor mediante potenciometría con electrodo de ión específico, siguiendo la técnica de calibración seriada y las de creatinina por la 
técnica de cromatografía de líquidos de alto rendimiento, utilizando una columna de fase reversa y con detección ultravioleta. Las determinaciones de cada variable se sometieron a sus respectivos controles de calidad (repeticiones ciegas, control de repetitividad, control de exactitud y control de precisión). Todos los análisis se realizaron en el laboratorio de bioquímica clínica de la Fundación Jiménez Díaz.

Para el análisis estadístico de los resultados se empleó la media y la desviación típica para las variables cuantitativas y porcentajes para las variables categóricas. En la estadística inferencial se utilizó el test $\mathrm{T}$ para las variables con distribuciones normales. En el caso de variables cuantitativas no normales, las comparaciones de medias entre grupos múltiples se realizaron con el test de Kruskall-Wallis. Para comparaciones entre dos determinaciones consecutivas en los mismos individuos se utilizó el test de Wilcoxon. El análisis estadístico se realizó con el paquete estadístico SPSS V.11.0.0.

\section{RESULTADOS}

La cantidad de pasta usada por la mayoría de los niños osciló entre 1.016 a 1.354 $\mathrm{mg}$, siendo la media $1.192 \mathrm{mg}$ (DT: 421).

El grupo de 5-6 años empleó una media de $1.064,9 \mathrm{mg}$ de dentífrico $(\mathrm{DT}=401,2) \mathrm{y}$ el de 7-8 años 1.398,8 mg (DT=370,6). La diferencia en la dosis de dentífrico entre los dos grupos de edad es estadísticamente significativa $(\mathrm{p}<0,001)$.

La tabla 1 recoge los valores medios de $\mathrm{pH}$, creatinina, flúor y $\mathrm{F} / \mathrm{Cr}$ en ambas muestras de orina, antes y después del cepillado en el grupo de estudio y en la tabla 2 aparecen los datos relativos a las dos muestras de orina del grupo de referencia (sin cepillado). En la tabla 3 se recogen los datos del

Tabla 1

Descriptivos de las variables pH, Creatinina, F y F/Cr en la $1^{\mathrm{a}}$ y $2^{\mathrm{a}}$ muestra de orina. Grupo de Estudio

\begin{tabular}{|c|c|c|c|c|c|c|c|c|c|}
\hline & $\mathbf{N}$ & $\begin{array}{c}\mathrm{pH} \\
1^{\mathrm{a}} \text { muestra }\end{array}$ & $\underset{2^{\mathrm{a}} \text { muestra }}{\mathrm{pH}}$ & $\begin{array}{c}\text { Creatinina } \\
1^{\text {a }} \text { muestra } \\
\mathrm{g} / \mathrm{l}\end{array}$ & $\begin{array}{c}\text { Creatinina } \\
2^{\mathrm{a}} \text { muestra } \\
\text { g/l }\end{array}$ & $\begin{array}{c}\text { Flúor } \\
1^{\mathrm{a}} \text { muestra } \\
\mathrm{mg} / \mathrm{l}\end{array}$ & $\begin{array}{c}\text { Flúor } \\
2^{\mathrm{a}} \text { muestra } \\
\mathrm{mg} / \mathrm{l}\end{array}$ & $\begin{array}{c}\mathrm{F} / \mathrm{Cr} \\
1^{\mathrm{a}} \text { muestra } \\
\mathrm{mg} / \mathrm{g}\end{array}$ & $\begin{array}{c}\mathrm{F} / \mathrm{Cr} \\
2^{\mathrm{a}} \mathrm{muestra} \\
\mathrm{mg} / \mathrm{g}\end{array}$ \\
\hline Media & 100 & 6,35 & 6,36 & 0,76 & 0,81 & 0,42 & 0,92 & 0,61 & 1,25 \\
\hline Mediana & 100 & 6,00 & 6,04 & 0,70 & 0,80 & 0,34 & 0,73 & 0,46 & 0,97 \\
\hline Moda & 100 & 5,85 & 5,36 & 0,80 & 0,80 & 0,23 & 0,50 & 0,60 & 0,90 \\
\hline D.T. & 100 & 1,08 & 1,08 & 0,30 & 0,35 & 0,31 & 0,75 & 0,51 & 0,93 \\
\hline Mínimo & 100 & 5,00 & 4,94 & 0,20 & 0,20 & 0,12 & 0,15 & 0,09 & 0,17 \\
\hline Máximo & 100 & 9,53 & 9,31 & 1,60 & 1,80 & 2,02 & 5,39 & 4,04 & 4,43 \\
\hline
\end{tabular}

Tabla 2

Descriptivos de las variables pH, Creatinina, F y F/Cr en la $1^{\mathrm{a}}$ y $2^{\mathrm{a}}$ muestra de orina. Grupo de Referencia

\begin{tabular}{|l|c|c|c|c|c|c|c|c|c|}
\hline & $\mathbf{N}$ & $\begin{array}{c}\mathbf{p H} \\
\mathbf{1}^{\mathbf{a}} \text { muestra }\end{array}$ & $\begin{array}{c}\mathbf{p H} \\
\mathbf{2}^{\mathbf{a}} \mathbf{m u e s t r a}\end{array}$ & $\begin{array}{c}\text { Creatinina } \\
\mathbf{1}^{\mathbf{a}} \text { muestra } \\
\mathbf{g} / \mathbf{l}\end{array}$ & $\begin{array}{c}\text { Creatinina } \\
\mathbf{2}^{\mathbf{a}} \text { muestra } \\
\mathbf{g} / \mathbf{l}\end{array}$ & $\begin{array}{c}\text { Flúor } \\
\mathbf{1}^{\mathbf{a}} \text { muestra } \\
\mathbf{m g} / \mathbf{l}\end{array}$ & $\begin{array}{c}\text { Flúor } \\
\mathbf{2}^{\mathbf{a}} \mathbf{m u e s t r a} \\
\mathbf{m g} / \mathbf{l}\end{array}$ & $\begin{array}{c}\mathbf{F} / \mathbf{C r} \\
\mathbf{1}^{\mathbf{a}} \mathbf{m u e s t r a} \\
\mathbf{m g} / \mathbf{g}\end{array}$ & $\begin{array}{c}\mathbf{F} / \mathbf{C r} \\
\mathbf{2}^{\mathbf{a}} \mathbf{m u e s t r a} \\
\mathbf{m g} / \mathbf{g}\end{array}$ \\
\hline Media & 15 & 5,83 & 5,94 & 0,75 & 0,70 & 0,35 & 0,28 & 0,45 & 0,43 \\
\hline Mediana & 15 & 5,67 & 5,76 & 0,70 & 0,70 & 0,29 & 0,27 & 0,47 & 0,43 \\
\hline Moda & 15 & 5,73 & 5,01 & 0,40 & 0,70 & 0,11 & 0,17 & 0,21 & 0,23 \\
\hline D.T. & 15 & 0,70 & 0,67 & 0,36 & 0,37 & 0,27 & 0,14 & 0,17 & 0,14 \\
\hline Mínimo & 15 & 4,99 & 5,01 & 0,40 & 0,10 & 0,11 & 0,08 & 0,21 & 0,23 \\
\hline Máximo & 15 & 7,44 & 7,26 & 1,60 & 1,60 & 1,18 & 0,62 & 0,74 & 0,80 \\
\hline
\end{tabular}


Tabla 3

Análisis de las comparaciones antes-después de pH, Creatinina, F, y F/Cr

\begin{tabular}{|c|c|c|c|c|}
\hline \multirow{2}{*}{ Diferencia } & \multicolumn{2}{|c|}{ Grupo Estudio } & \multicolumn{2}{|c|}{ Grupo Referencia } \\
\hline & Valor & $\mathbf{p}$ & Valor & $\mathbf{p}$ \\
\hline $\mathrm{pH} 2^{\mathrm{a}}$ muestra - $\mathrm{pH} 1^{\mathrm{a}}$ muestra & 0,01 & 0,985 & 0,11 & 0,589 \\
\hline Creatinina $2^{\mathrm{a}}$ muestra - Creatinina $1^{\mathrm{a}}$ muestra & $0,05 \mathrm{~g} / \mathrm{l}$ & 0,125 & $-0,05 \mathrm{~g} / \mathrm{l}$ & 0,787 \\
\hline Flúor $2^{\mathrm{a}}$ muestra - Flúor $1^{\mathrm{a}}$ muestra & $0,50 \mathrm{mg} / \mathrm{l}$ & $<0,001$ & $-0,07 \mathrm{mg} / \mathrm{l}$ & 0,209 \\
\hline $\mathrm{F} / \mathrm{Cr} 2^{\mathrm{a}}$ muestra $-\mathrm{F} / \mathrm{Cr} 1^{\mathrm{a}}$ muestra & $0,64 \mathrm{mg} / \mathrm{g}$ & $<0,001$ & $-0,02 \mathrm{mg} / \mathrm{g}$ & 0,427 \\
\hline
\end{tabular}

Prueba de rangos con signo de Wilcoxon.

Tabla 4

Relación entre la dosis de dentífrico y las variables pH, Creatinina, F y F/Cr

\begin{tabular}{|l|c|c|c|c|}
\hline & pH 2 $2^{\text {a }}$ muestra & Creatinina 2 $\mathbf{2}^{\mathbf{a}}$ muestra & Flúor 2 ${ }^{\mathbf{a}}$ muestra & F/Cr 2 ${ }^{\mathbf{a}}$ muestra \\
\hline chi-cuadrado & 8,53 & 10,98 & 42,64 & 37,54 \\
\hline Significación asintótica & 0,188 & 0,089 & $<0,001$ & $<0,001$ \\
\hline
\end{tabular}

Tabla 5

Cantidad de dentífrico por cepillado

\begin{tabular}{|c|c|c|c|}
\hline Autor & Edad & Cantidad dentífrico & Año \\
\hline Bruun y Thylstrup & 7 años & $1.500 \mathrm{mg}$ & 1988 \\
\hline \multirow{2}{*}{ Dowell } & $>3$ años & $1.000 \mathrm{mg}$ & \multirow{2}{*}{1981} \\
\hline & $<3$ años & $540 \mathrm{mg}$ & \\
\hline Baxter & 5-16 años & $990 \mathrm{mg}$ & 1980 \\
\hline \multirow{3}{*}{ Barnard et als } & 2-4 años & $860 \mathrm{mg}$ & \multirow{3}{*}{1974} \\
\hline & 5-7 años & $940 \mathrm{mg}$ & \\
\hline & 11-13 años & $1.100 \mathrm{mg}$ & \\
\hline Ericsson y Forsman & 4-7 años & $400 \mathrm{mg}$ & 1969 \\
\hline
\end{tabular}

análisis de los cambios producidos en las distintas variables en la segunda muestra de orina con respecto a la $1^{\mathrm{a}}$ en ambos grupos. En el grupo de estudio no se apreciaron diferencias en los valores de $\mathrm{pH}$ ni de creatinina entre la $1^{\mathrm{a}}$ y la $2^{\mathrm{a}}$ muestra $(\mathrm{p}>0,05) \mathrm{y}$, por el contrario, sí fueron significativas las diferencias en la concentración de flúor y $\mathrm{F} / \mathrm{Cr}$ entre ambas muestras ( $<<0,001)$. En el grupo de referencia no se observaron diferencias significativas entre las dos muestras de orina para ninguna de las variables estudiadas $(\mathrm{P}>0,05)$.

Se analizó la posible influencia de la variable "dosis de dentífrico" sobre los valores de $\mathrm{pH}$, creatinina y flúor en orina, registrados en la $2^{\mathrm{a}}$ muestra, constatándose que al aumentar la dosis de dentífrico,
Figura 1

Valores medios de $\mathrm{F} / \mathrm{Cr}$ en la $\mathbf{1}^{\mathrm{a}}$ muestra de orina según la dosis de dentífrico utilizada

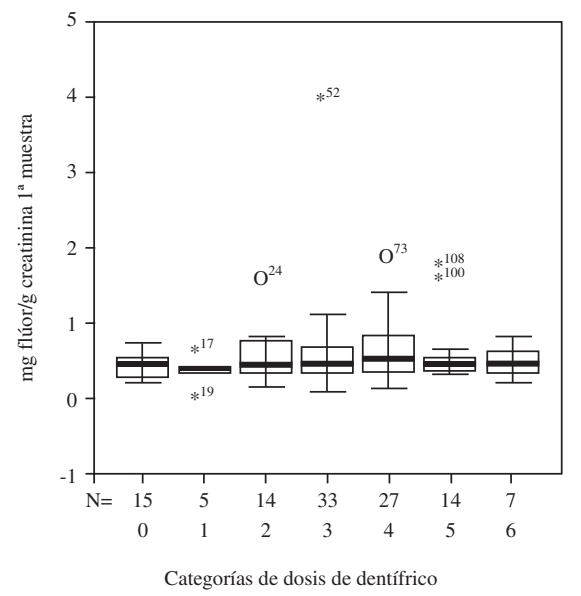

Rev Esp Salud Pública 2009, Vol. 83, N. 3 
Figura 2

Valores medios de $\mathrm{F} / \mathrm{Cr}$ en la $2^{\mathrm{a}}$ muestra de orina según la dosis de dentífrico utilizada

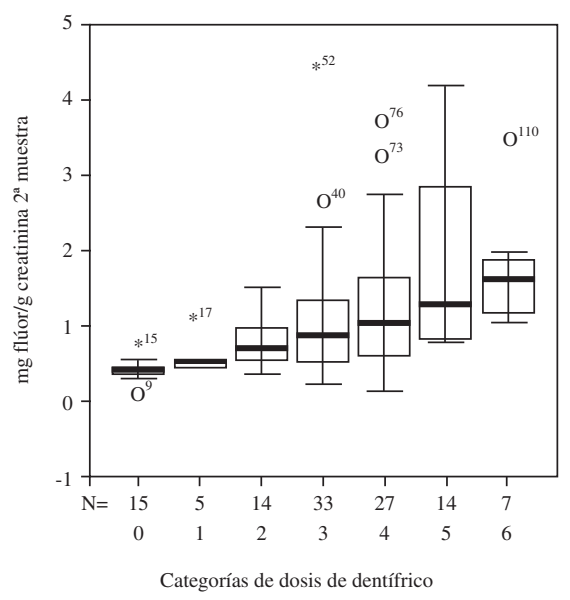

aumentaba proporcionalmente la concentración de flúor en orina $(\mathrm{p}<0,001) \mathrm{y}$, como consecuencia de ello, también aumentaba la razón $\mathrm{F} / \mathrm{Cr}(\mathrm{p}<0,001)$. En cambio, el aumento de la dosis de dentífrico no influyó sobre los valores de $\mathrm{pH}$ y la concentración de creatinina $(\mathrm{P}>0,05)$ (tabla 4$)$.

En la figura 1 se representan los valores medios de $\mathrm{F} / \mathrm{Cr}$ obtenidos en la $1^{\mathrm{a}}$ muestra de orina en cada una de las seis categorías de sujetos del grupo de estudio, en función de la dosis de dentífrico utilizado, y en el grupo de referencia, cuya dosis de dentífrico era 0 . En la figura 2 se representan los valores correspondientes a la $2^{\mathrm{a}}$ muestra de orina. La representación mediante boxplot evidencia una tendencia ascendente de los niveles de excreción de flúor en orina, relacionada con la dosis de dentífrico empleada. Las diferencias de los valores de entre la $1^{\mathrm{a}}$ y $2^{\mathrm{a}}$ muestra de orina del grupo de referencia y de las 6 categorías del grupo de estudio, en función de las dosis de dentífrico, aparecen en la figura 3. Como puede observarse, los valores son superiores en la $2^{\mathrm{a}}$ muestra en todas las categorías del grupo de estudio y no así en el grupo de referencia.
Figura 3

Variación de $\mathrm{F} / \mathrm{Cr}$ entre la $1^{\mathrm{a}}$ y la $2^{\mathrm{a}}$ muestra de orina en función de la dosis de dentífrico

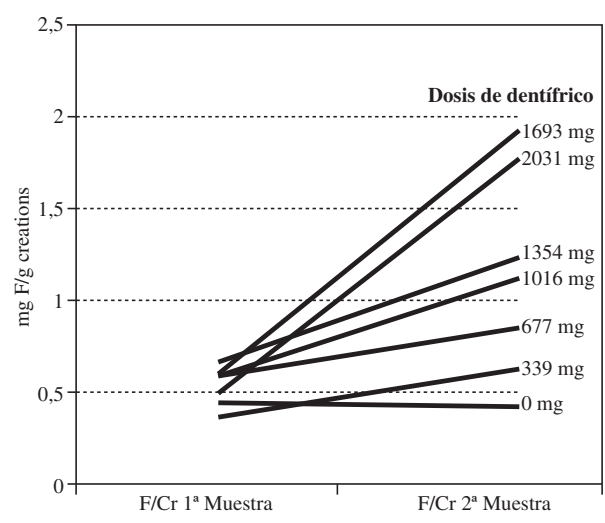

\section{DISCUSIÓN}

La cantidad media de pasta dental utilizada por los niños es superior a la referida en la mayoría de los estudios publica$\operatorname{dos}^{32-34}$, aunque coincide con varios de ellos en que el peso medio de dentífrico utilizado es proporcional a la edad de los niños ${ }^{33}$. En la tabla 5 se reflejan los valores obtenidos por otros autores sobre la cantidad de pasta autoadministrada por los niños ${ }^{32-36}$.

El contenido de FNa de la pasta utilizada en nuestro estudio era $1.100 \mathrm{ppm}$. Teniendo en cuenta esa proporción, 1.192 mg de pasta -cantidad media utilizada por los niños en cada cepillado- incluirían aproximadamente $1,31 \mathrm{mg}$ de $\mathrm{FNa}$, de los que 0,66 mg corresponderían a ion flúor. Esta cantidad de flúor, que entraría en la boca del niño en cada cepillado, tendría que multiplicarse por el número de cepillados realizados al día. Un niño de nuestra muestra que se cepillara los dientes tres veces al día se estaría introduciendo en la boca casi $4 \mathrm{mg}$ de FNa y por tanto aproximadamente $2 \mathrm{mg}$ de ion flúor. Se ha señalado que los niños pueden llegar a deglutir entre el 25 y el $33 \%$ del dentífrico utiliza$\mathrm{do}^{37}$, dependiendo de varios factores 
(edad, sabor del dentífrico, enjuague posterior al cepillado, etc) ${ }^{38}$. Eso supone que los niños de nuestra muestra podrían deglutir en cada cepillado entre 0,5 y 0,66 $\mathrm{mg}$ de ion flúor.

El hecho de que se utilice una cantidad excesiva de pasta en cada cepillado puede atribuirse a falta de educación sanitaria en los niños y sus padres junto con otros factores $^{38}$ : El aspecto y sabor agradables del producto pueden incentivar su ingesta, a lo que se une la influencia de los mensajes publicitarios que incitan a la utilización de dosis elevadas de dentífrico. Así mismo, pueden influir la longitud del cabezal del cepillo, el diámetro del orificio de salida del tubo de pasta y la facilidad de extracción de su contenido.

En cuanto a la medición de flúor en orina en este estudio se optó por utilizar muestras de orina puntuales, recogidas en el momento en que se esperaba encontrar el pico más alto de excreción, aproximadamente 2 horas después del cepillado de dientes con un dentífrico fluorado ${ }^{24}$. Los resultados de la determinación de las concentraciones de flúor se expresaron mediante la razón flúor/creatinina $(\mathrm{F} / \mathrm{Cr})^{27}$. En otros trabajos publicados para la medición de flúor en orina se utiliza habitualmente la excreción total en orina de 24 horas26. En este estudio, esta modalidad de cuantificación se rechazó esta modalidad de cuantificación se rechazó por dos motivos: Por una parte debido a la dificultad inherente a realizar el seguimiento en niños tan pequeños que acudían diariamente a la escuela. Por otra parte, aunque la orina de 24 horas permite conocer la excreción total del flúor durante este periodo de tiempo, no refleja con precisión el aumento en su excreción como consecuencia de la introducción de un factor de riesgo, que en este caso es el dentífrico, pues no es útil para distinguir las elevaciones de los niveles de flúor debidas a otros factores,como, por ejemplo, la dieta. En cuanto al $\mathrm{pH}$ de la orina y su concentración de creatinina, los valores de creatinina de la $1^{\mathrm{a}}$ muestra obtenida antes del cepillado se encontraban en los límites normales y se mantuvieron prácticamente constantes en la $2^{\text {a }}$ muestra,obtenida después del mismo. Este hallazgo era esperable teniendo en cuenta que entre la obtención de ambas muestras no intervino ningún factor, como la ingesta de alimentos o un incremento de la actividad física, que pudiera ejercer un efecto significativo sobre esos dos parámetros.

En este estudio, la concentración de flúor en la orina de los niños antes del cepillado fue $0,42 \mathrm{mg} / \mathrm{l}$, una cifra igual a la referida por Shanon y Sanders ${ }^{39}$ que analizaron muestras puntuales de orina en un grupo de niños de 6-9 años, residentes en una comunidad con aguas no fluoradas. Por el contrario, la concentración encontrada por ObryMusset et $\mathrm{al}^{40}$ en una población de niños de 10-14 años que no recibían suplementos de flúor fue muy inferior $(0,28 \mathrm{mg} / \mathrm{l})$. Estos autores realizaron sus análisis en muestras de orina de 24 horas.

Al no encontrar otros trabajos con una metodología similar a la nuestra, hemos comparado nuestros hallazgos con los de otras investigaciones en las que se analiza el flúor en la orina de individuos que reciben suplementos fluorados por vía oral, ya sea aguas fluoradas o tabletas de flúor.

Así, Baez ${ }^{41}$ estudió la concentración de flúor en 3 muestras de orina recogidas a lo largo de 24 horas en un grupo de niños de 4 a 6 años que residían en una comunidad con aguas fluoradas, encontrando concentraciones medias de día y de noche de 1,26 y 1,42 $\mathrm{mg} / \mathrm{l}$, respectivamente. Por su parte RuggGunn et $\mathrm{al}^{42}$, en muestras de orina de 24 horas obtenidas en niños de 4 años de Sri Lanka y de Inglaterra que bebían habitualmente aguas fluoradas, encontraron respectivamente concentraciones de flúor de 1,09 mg/l y 0,94 mg/l. En la investigación antes mencionada de Obry-Musset et $\mathrm{al}^{40}$ se encontraron concentraciones de flúor de $0,99 \mathrm{mg} / \mathrm{l}$. en orina de 24 horas tras la admi- 
nistración de una tableta diaria de flúor de 1 mg. Estas diferencias en cuanto a excreción de flúor registradas en distintos estudios pueden atribuirse al contacto con flúor antes de la obtención de la muestra, bien vehiculado a través de enjuagues o dentífricos, o a través de la dieta.

En nuestro estudio, la concentración de flúor en las muestras de orina obtenida tras el cepillado experimentó un importante aumento $(0,92 \mathrm{mg} / \mathrm{l}, \mathrm{DT}=0,75$ frente a 0,42 $\mathrm{mg} / \mathrm{l}, \mathrm{DT}=0,34, \mathrm{p}<0,001)$, que resultaba directamente proporcional a la cantidad de dentífrico utilizada.

También aumentó significativamente la razón $\mathrm{F} / \mathrm{Cr}$ en la $2^{\mathrm{a}}$ muestra de orina con respecto a la $1^{\mathrm{a}}(1,25 \mathrm{mg}, \mathrm{DT}=0,93$ frente a $0,61 \mathrm{mg}, \mathrm{DT}=0,51, \mathrm{p}<0,001)$.

Los valores de $\mathrm{F} / \mathrm{Cr}$ obtenidos en la $2^{\mathrm{a}}$ muestra de orina son comparables a los citados por Declercq ${ }^{43}$. Este autor estudió tres grupos de niños menores de 14 años. En el primer grupo, que vivía cerca de una fábrica de aluminio en un entorno contaminado de flúor, el nivel medio de $\mathrm{F} / \mathrm{Cr}$ fue $0,52 \mathrm{mg} / \mathrm{g}$. La cifra correspondiente en el $2^{\circ}$ grupo de niños que vivía en una comunidad con aguas fluoradas fue $0,69 \mathrm{mg} / \mathrm{g}$, y en un tercer grupo, al que se administró diariamente una tableta fluorada, el nivel fue 0,82 $\mathrm{mg} / \mathrm{g}$. Por su parte Kertesz et al, analizando muestras puntuales de orina en niños de 813 años que bebían aguas fluoradas, encontraron una media de $1,51 \mathrm{mg} / \mathrm{g}^{27}$ y Zohouri et al en niños de 32 meses de edad que bebían asimismo aguas fluoradas, obtuvieron una media de $1,49 \mathrm{mg} / \mathrm{g}^{30}$.

En investigaciones que se realizan con muestras puntuales de orina no hemos encontrado estudios sobre la ingestión de dentífrico que relacionen el flúor excretado con la creatinina, lo que es muy habitual en estudios epidemiológicos con otros objeti$\operatorname{vos}^{44,45}$.
En nuestro estudio, el aumento observado en la razón $\mathrm{F} / \mathrm{Cr}$ en la $2^{\mathrm{a}}$ muestra de orina con respecto a la $1^{\mathrm{a}}$ era muy significativo $(\mathrm{p}<0,001) \mathrm{y}$, además, directamente proporcional a la cantidad de dentífrico utilizada. $(\mathrm{p}<0,001)$. El incremento en la concentración de flúor en orina tras el cepillado dental pone de manifiesto que el flúor vehiculado en el dentífrico que es introducido en la boca en cada cepillado penetra de algún modo a nivel sistémico, presumiblemente por ingestión ${ }^{12,13}$, aunque no se puede descartar su eventual absorción en mayor o menor grado a través de la mucosa. Está ampliamente demostrado que el efecto preventivo del flúor que transita por vía sistémica es mínimo y sin embargo es la principal causa de fluorosis dental cuando actúa en niños cuyo desarrollo dentario esta en fase de calcificación ${ }^{23}$, además de comportar otros posibles efectos indeseables, tales como intoxicaciones agudas.

Los resultados de este trabajo indican que las concentraciones de flúor en orina como expresión de la penetración sistémica del mismo son proporcionales a la cantidad de dentífrico empleado y alertan sobre el uso de una cantidad excesiva de dentífrico por los niños que se autoadministran la pasta sin el control de un adulto. Por tanto una forma de prevenir los posibles riesgos vinculados a la exposición excesiva al flúor a nivel sistémico es controlar la cantidad de dentífrico utilizada por el niño. Para ello hay que propiciar la educación sanitaria de padres, pediatras y, en su caso, maestros y cuidadores infantiles, para que conozcan qué cantidad de dentífrico deben utilizar los niños de distintas edades para su higiene dental habitual y a qué edad pueden comenzar a administrarse la pasta dental por sí mismos.

\section{BIBLIOGRAFÍA}

1. Bravo M, Casals E, Cortés FJ, Llodra JC. Encuesta de Salud Oral en España 2005. RCOE 2006;11(4):409-456. 
2. Nithila A, Bourgeois D, Barmes DE, Murtomaa H. WHO Global Oral Data Bank, 1986-96: an overview of oral health surveys at 12 years of age. Bull World Health Organ. 1998;76(3):237-44.

3. García-Camba JM, Oñorbe M. La salud bucodental de la población infantil en la Comunidad de Madrid. Comunidad de Madrid. Consejería de Salud. Documentos técnicos de salud pública $\mathrm{N}^{\mathrm{o}}$ 3. $2^{\mathrm{a}}$ ed.1994.

4. Ripa L. A half-century of community water fluoridation in the United States: review and commentary. J Public Health Dent. 1993;53:17-44.

5. Kumar JV, Moss ME. Fluorides in dental public health programs. Dent Clin North Am. 2008;52 (2):387-401.

6. Topping G, Assaf A. Strong evidence that daily use of fluoride toothpaste prevents caries. Acta Odontol Scand. 2003;61(6):347-55.

7. Limeback H, Ismail A, Banting D, DenBesten P, Featherstone J, Riordan PJ. Canadian Consensus Conference on the appropriate use of fluoride supplements for the prevention of dental caries in children. J Can Dent Assoc. 1998;64(9):636-9.

8. Burt BA, Eklund SA. The methods of oral epidemiology. In: Burt B, Eklund S. Dentistry dental practice, and the community. 5th ed. W.B.Saunders Company. Philadelphia. 1999:159-99.

9. Pendrys DG. The epidemiology of oral diseases. In: Gluck MG, Morganstein WM. Jong's community dental health. 4th ed. St Louis, Missouri. Mosby. 1998:121-43.

10. Fomon S, Ekstrand J. Fluoride intake by infants. J Public Health Dent 1999;59(4):229-34.

11. Rojas-Sanchez F, Kelly SA, Drake KM, Eckert GJ, Stookey GK, Dunipace AJ. Fluoride intake from foods, beverages and dentifrice by young children in communities with negligibly and optimally fluoridated water: a pilot study. Community Dent Oral Epidemiol 1999;27:287-97.

12. van Loveren C, Ketley CE, Cochran JA, Duckworth RM, O'Mullane DM. Fluoride ingestion from toothpaste: fluoride recovered from the toothbrush, the expectorate and the after-brush rinses. Community Dent Oral Epidemiol. 2004;32 Suppl 1:54-61.

13. de Almeida BS, da Silva Cardoso VE, Buzalaf MA. Fluoride ingestion from toothpaste and diet in 1- to 3-year-old Brazilian children. Community Dent Oral Epidemiol. 2007;35(1):53-63.
14. Maguire A, Zohouri FV, Hindmarch PN, Hatts J, Moynihan PJ. Fluoride intake and urinary excretion in 6- to 7-year-old children living in optimally, sub-optimally and non-fluoridated areas. Community Dent Oral Epidemiol 2007; 35(6): $479-88$

15. Moraes SM, Pessan JP, Ramires I, Buzalaf MA. Fluoride intake from regular and low fluoride dentifrices by 2-3-year-old children: influence of the dentifrice flavor. Braz Oral Res. 2007;21(3):23440.

16. Limeback H. A re-examination of the pre-eruptive and post-eruptive mechanism of the anti-caries effects of fluoride: is there any anti-caries benefit from swallowing fluoride? Community Dent Oral Epidemiol. 1999;27(1):62-71.

17. Whitford GM. Fluoride metabolism and excretion in children. J Public Health Dent. 1999;59 (4):224-8.

18. Whitford GM. Intake and metabolism of fluoride. Adv Dent Res. 1994;8(1):5-14.

19. González A, Gil J, Gil C, Algar J, Alos L, Rosado J. Bases para el uso racional del flúor en la prevención y tratamiento de caries en pediatría. Rev Pediatría de Atención Primaria. 1999;1(2):93-111.

20. Cremer HD, Buttner W. Absorción de los fluoruros. En: Fluoruros y salud. OMS. Ginebra. 1972: 75-91.

21. Ramos JA. Bioquímica dental. Síntesis S.A. Madrid 1996:105-13.

22. Ekstrand J, Whitford GM. Fluoride metabolisme. In: Ekstrand J, Fejerskov O, Silverstone. Fluoride in dentistry. Munksgaard. Copenhagen. 1988:150-70.

23. Whitford GM. Determinants and mechanisms of enamel fluorosis. Ciba Found Symp, 1997; 205:226-41.

24. Bell ME, Largent EJ, Ludwig TG, Muhler JC, Stookey GK. El aporte de flúor al hombre. En: Fluoruros y salud. OMS. Ginebra. 1972: 17-73.

25. Axelsson P. Other caries-preventive factors. In: Axelsson P. An introduction to risk prediction and preventive dentistry. Germany- Quintessence books; 1999:77-103

26. Murray JJ. Occurrence and metabolism of fluorides. In: Murray JJ. Appropriate use of fluorides for human health. In: World Health Organization. Geneva. 1986: 3-32. 
27. Kertesz P, Bánócky J, Ritlop B, Beródy A, Péter $\mathrm{M}$. The determination of urinary fluoride/ creatinina ratio $(\mathrm{Q})$ in monitoring fluoride intake. Acta Physiol Hung. 1989;74(3-4):209-14

28 Castro S. Riñón y vías urinarias. En: Castro S. Manual de patología general. Masson $5^{\mathrm{a}}$ ed. Barcelona. 1996: 275-313.

29. Rose BD, Post TW. Renal circulation and glomerular filtration rate. In: Rose BD, Post TW. McGraw-Hill. 2001;21-70.

30. Zohouri FV, Swinbank CM, Maguire A, Moynihan PJ. Is the fluoride/creatinine ratio of a spot urine sample indicative of 24-h urinary fluoride? Community Dent Oral Epidemiol. 2006; 34: 130-8.

31. Székely M, Bánóczy J, Fazakas Z, Hobai S, Villa A. A comparison of two methods for the evaluation of the daily urinary fluoride excretion in Romanian pre-school children. Community Dent Health. 2008;25(1):23-7.

32. Baxter PM. Toothpaste ingestion during toothbrushing by school children. Brit Dent 1980;148 (5): $125-8$

33. Barnhart WE, Hiller LK, Leonard GJ, Michaels SE. Dentifrice usage and ingestion among four age groups. J Dent Res. 1974;53(6):1317-22.

34. Bruun C, Thylstrup A. Dentifrice usage among Danish children. J Dent Res. 1988;67(8):1114-7

35. Ericsson Y, Forsman B. Fluoride retained from mouthrinses and dentifrices in preschool children. Caries Res. 1969;3(3):290-9.

36. Dowell TB. The use of toothpaste in infancy. $\mathrm{Br}$ Dent J. 1981;150(9):247-9.

37. Dincer E. Why do I have white spots on my front teeth? N Y State Dent J. 2008;74(1):58-60.
38. Naccache H, Simard PL, Trahan L, Brodeur JM, Demers M, Lachapelle D, Bernard PM. Factors affecting the ingestion of fluoride dentifrice by children. J Public Health Dent. 1992; 52(4): 222-6.

39. Shannon IL, Sanders DM. Urinary fluoride concentration in 6- to 9-year-old children drinking water with a very low fluoride level. Caries Res. 1979;13(1):18-22.

40. Obry-Musset AM, Bettembourg D, Cahen PM, Voegel JC, Frank RM. Urinary fluoride excretion in children using potassium fluoride containing salt or sodium fluoride supplements. Caries Res 1992;26 (5):367-70.

41. Baez RJ, Baez MX, Marthaler TM. Urinary fluoride excretion by children 4-6 years old in a south Texas community. Rev Panam Salud Publica 2000;7(4):242-8.

42. Rugg-Gunn AJ, Nunn JH, Ekanayake L, Saparamadu KD, Wright WG. Urinary fluoride excretion in 4-year-old children in Sri Lanka and England. Caries Res 1993;27(6):478-83.

43. Declercq C, Ponti P, Warembourg D, Tronet V, Rousselle JF. Urinary excretion of fluorides in children living around an aluminum smelter. Rev Epidemiol Sante Publique 1995; 43(5): 504-9.

44. Xiang Q, Liang Y, Chen L, Wang C, Chen B Chen $\mathrm{X}$, Zhou M. Effect of fluoride in drinking water on children's intelligence. Fluoride 2003; 36(2): 84-94.

45. Conzen P, Nuscheler M, Melotte A, Verhaegen M, Leupolt T, Van Aken, H, Peter K. Renal function and serum fluoride concentrations in patients with stable renal insufficiency after anesthesia with sevoflurane or enflurane. Anesthesia \& Analgesia 1995;81(3):569-575. 
